\title{
Rescuing the concept of swimming in curved spacetime
}

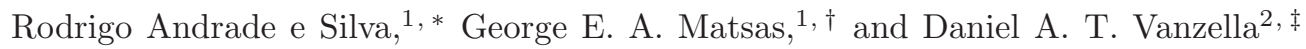 \\ ${ }^{1}$ Instituto de Física Teórica, Universidade Estadual Paulista, \\ Rua Dr. Bento Teobaldo Ferraz, 271 - Bl. II, 01140-070, São Paulo, SP, Brazil \\ ${ }^{2}$ Instituto de Física de São Carlos, Universidade de São Paulo, \\ Cx. Postal 369, 13560-970, São Carlos, SP, Brazil
}

(Dated: November 21, 2016)

\begin{abstract}
It has been argued that an extended, quasi-rigid body evolving freely in curved spacetime can deviate from its natural trajectory by simply performing cyclic deformations. More interestingly, in the limit of rapid cycles, the amount of deviation, per cycle, would depend on the sequence of deformations but not on how fast they are performed - like the motion of a swimmer at low Reynolds number. Here, however, we show that the original analysis which supported this idea is inappropriate to investigate the motion of extended bodies in the context of general relativity, rendering its quantitative results invalid and casting doubts on the reality of this swimming effect. We illustrate this by showing that the original analysis leads to a non-zero deviation even in a scenario where no swimming can possibly occur. Notwithstanding, by applying a fully covariant, local formalism, we show that swimming in curved spacetime is indeed possible and that, in general, its magnitude can be of the same order as (fortuitously) anticipated - although it is highly suppressed in the particular scenario where it was originally investigated.
\end{abstract}

PACS numbers: 04.20.-q

\section{INTRODUCTION}

More than a hundred years have passed and the picture of reality provided by special and general relativity can still surprise (and sometimes deceive) our Newtonian intuition built upon notions of absolute space and time. In an analysis published in 2003 [1], J. Wisdom concluded that an extended, quasi-rigid body, free from external non-gravitational forces, could propel itself through curved space by performing cyclic internal motions. In the limit of rapid cycles, the amount of spatial translation (or, more properly, deviation from the trajectory it would follow if rigid) per cycle would depend on the sequence of conformational changes of the body but not on how fast they are performed, similarly to the motion of a swimmer at low Reynolds number - hence the term swimming effect. This would be a legitimate curved-space effect, absent in Newtonian gravity, where this deviation goes to zero in the limit of rapid cycles (as stated in Ref. [2] in response to Ref. [3]).

However, as novel and appealing as Wisdom's idea may be, here we show that his original analysis is inappropriate to investigate the motion of extended bodies in curved spacetimes. We illustrate this by repeating his analysis in a scenario where no swimming can possibly occur and show that it leads to a non-zero, fictitious effect, which renders his previous quantitative results invalid and casts serious doubts on the reality of the swimming effect. Notwithstanding, coming to the rescue of

\footnotetext{
*Electronic address: rasilva@ift.unesp.br

${ }^{\dagger}$ Electronic address: matsas@ift.unesp.br

${ }^{\ddagger}$ Electronic address: vanzella@ifsc.usp.br
}

Wisdom's idea, we use a covariant, local approach developed in the 1970's by W. G. Dixon [4 7] to show that swimming in curved spacetime is indeed possible and argue that, in general, it can be of the same order of magnitude as the (fictitious) effect obtained in Ref. [1].

Our paper is organized as follows. In Sec. III we recall Wisdom's result for an articulated tripod evolving in Schwarzschild spacetime and apply the same approach to a tripod in de Sitter spacetime. In this latter context, we obtain a nonzero net displacement for the tripod which is very similar to the one obtained in the former case. However, in Sec. III] after presenting a summary of Dixon's formalism, we show that no swimming can possibly occur in maximally-symmetric spacetimes, contradicting the result of the previous section and thus proving Wisdom's approch inappropriate. In Sec. IV] we revisit Wisdom's tripod in Schwarzschild spacetime using Dixon's formalism and show that, although possible, swimming in this case is highly suppressed in comparison to Wisdom's result. Finally, in Sec. V we make some final remarks, rescuing the curved-spacetime swimming effect in the general context.

\section{FICTITIOUS SPACETIME SWIMMING?}

For concreteness sake, we shall reuse Wisdom's symmetric tripod made of four point particles, one with mass $m_{0}$ at the vertex of the tripod and three with mass $m_{1}$, one at the end of each leg. Aligning the tripod symmetry axis along the radial direction of a spherically-symmetric object with mass $M$, and changing the length $l$ of its legs (measured by static observers) and the angle $\alpha$ between each leg and the symmetry axis (measured in a local sta- 
tionary Lorentz frame) according to the cycle

$$
\begin{aligned}
(l, \alpha)= & \left(l_{0}, \alpha_{0}\right) \rightarrow\left(l_{0}+\delta l, \alpha_{0}\right) \rightarrow\left(l_{0}+\delta l, \alpha_{0}+\delta \alpha\right) \\
& \rightarrow\left(l_{0}, \alpha_{0}+\delta \alpha\right) \rightarrow\left(l_{0}, \alpha_{0}\right)
\end{aligned}
$$

Wisdom calculated a radial deviation (with respect to the motion it would have if rigid), per cycle, to be given by

$$
\delta r_{0} \approx-\frac{3 m_{0} m_{1}}{\left(m_{0}+3 m_{1}\right)^{2}}\left(\frac{l_{0}}{r_{0}}\right)^{2} \frac{G M}{c^{2} r_{0}} \sin \alpha_{0} \delta l \delta \alpha,
$$

where $r_{0}$ is the radial coordinate of the mass $m_{0}$ ( $G$ is Newton's constant and $c$ is the speed of light). The result above would supposedly hold in the limit of rapid cycles (short periods - more on this, later), small velocities (in comparison to $c$ ), and $l_{0} / r_{0}, G M /\left(c^{2} r_{0}\right), \delta l / l_{0}, \delta \alpha / \alpha_{0} \ll$ 1 .

Now, let us consider this same tripod executing the same cycle but evolving in de Sitter spacetime - a maximally-symmetric spacetime with positive curvature. In order to avoid subtleties involving time-dependent metric components, we cover (a portion of) the spacetime with static coordinates, in which the line element takes the form

$$
d s^{2}=-\left(1-\frac{r^{2}}{\kappa^{2}}\right) c^{2} d t^{2}+\frac{d r^{2}}{\left(1-r^{2} / \kappa^{2}\right)}+r^{2} d \Omega^{2},
$$

where $\kappa$ is a constant related to the spacetime curvature $R=12 / \kappa^{2}, r \in[0, \kappa)$ is the radial coordinate with respect to some arbitrary spatial point, $t$ is the time-like coordinate, and $d \Omega^{2}=d \theta^{2}+(\sin \theta)^{2} d \phi^{2}$ is the line element of the unit sphere. We conveniently orient the tripod symmetry axis along the radial direction $\theta=0$, in such a way that its legs have constant $\phi$ coordinates (for instance, $\phi=0,2 \pi / 3,4 \pi / 3)$.

Following the reasoning of Ref. [1], the Lagrangian of the tripod would be the sum of the Lagrangians of each particle, supplemented by the constraints which relate the position of the particles in terms of $l(t)$ and $\alpha(t)$ :

$$
\begin{aligned}
L\left(r_{0}, \dot{r}_{0}, t\right)= & -m_{0} c^{2} \sqrt{\left(1-\frac{r_{0}^{2}}{\kappa^{2}}\right)-\frac{\dot{r}_{0}^{2} / c^{2}}{\left(1-\frac{r_{0}^{2}}{\kappa^{2}}\right)}} \\
& -3 m_{1} c^{2} \sqrt{\left(1-\frac{r_{1}^{2}}{\kappa^{2}}\right)-\frac{\dot{r}_{1}^{2} / c^{2}}{\left(1-\frac{r_{1}^{2}}{\kappa^{2}}\right)}-\frac{r_{1}^{2} \dot{\theta}_{1}^{2}}{c^{2}}}
\end{aligned}
$$

where dots represent derivative with respect to $t$. Note that the total Lagrangian is a function of $\left(r_{0}, \dot{r}_{0}\right)$, associated with the motion of the vertex of the tripod, and of the assigned functions $l(t)$ and $\alpha(t)$, which relate the positions of the other three particles, at $\left(r_{1}, \theta_{1}, \phi=0\right)$, $\left(r_{1}, \theta_{1}, \phi=2 \pi / 3\right),\left(r_{1}, \theta_{1}, \phi=4 \pi / 3\right)$, with $r_{0}$. Going through all the steps which led Wisdom to Eq. (2), we obtain, for the same cycle (1),

$$
\delta r_{0} \approx \frac{3 m_{0} m_{1}}{\left(m_{0}+3 m_{1}\right)^{2}}\left(\frac{l_{0}}{\kappa}\right)^{2} \sin \alpha_{0} \delta l \delta \alpha .
$$

This result is, both qualitatively and quantitatively, very similar to Eq. (2), with the scale of curvature of Schwarzschild spacetime, $G M /\left(c^{2} r^{3}\right)$, replaced by the scale of curvature of de Sitter spacetime, $1 / \kappa^{2}$ (the change in sign is due to the fact that "free fall" in de Sitter is towards increasing values of $r$ ). In fact, the results are so similar and consistent with each other that it is difficult to raise any objection to one of them without compromising the other. But, as we argue below, the deviation obtained in Eq. (5) is entirely fictitious.

\section{NO-SWIMMING IN MAXIMALLY-SYMMETRIC SPACETIMES}

The motion of extended bodies in general relativity was analyzed in detail, in a manifestly covariant and local way, in a seminal series of papers by W. G. Dixon in the 1970's [4 7]. One great advantage of Dixon's formalism, in addition to being local and covariant, is that covariant conservation of the stress-energy-momentum tensor of the body - which strictly implements the "absence of external non-gravitational forces" in curved spacetime can be enforced from the beginning [8]. Here we shall collect only the results which are relevant to our covariant analysis of the swimming effect, focusing on their physical meaning and implications rather than on technical definitions, and refer the reader to the original papers for further details. (Some technical remarks whose omission we may find unbearable we include as endnotes.)

Let $T^{a b}$ be the stress-energy-momentum tensor of the body under consideration. Its ten independent components describe, at each point, the energy and momentum densities of the body, as well as local internal forces exchanged among its parts. We assume, from the beginning, that $T^{a b}$ is covariantly conserved: $\nabla_{a} T^{a b}=0$, where $\nabla_{a}$ stands for the covariant derivative compatible with the spacetime geometry. Thus, under reasonable assumptions about $T^{a b}$ [9] and somewhat weak conditions on the "strength" of the gravitational field (see Sec. 2 of Ref. [10]), the following general results hold:

(i) At each and every spacetime point $x$ in the worldtube of the body (i.e., at which $T^{a b}(x) \neq 0$ ), there is a unique time-like, future-pointing, unit vector $n^{a}=n^{a}(x)$ such that the total four-momentum $p^{a}=p^{a}(x, n)$ of the body (properly defined with respect to $x$ and $n^{a}$; see Ref. [4] ) is entirely in the direction of $n^{a}$. In essence, this means that there is a family of observers (those with four-velocity $u^{a}=c n^{a}$ ) according to whom the total spatial momentum of the body is zero;

(ii) There is a unique, covariantly defined, time-like curve $z(\tau)$, contained in the (convex hull of the) world-tube of the body, which can be consistently identified as the world-line of its center of mass (here $\tau$ is the proper-time along this world-line). It is worth mentioning that the tangent vector to 
this curve, $v^{a}(\tau)$, is not, in general, parallel to the four-velocity $u^{a}=c n^{a}-$ see $(\mathrm{i})$ - at $z(\tau)$. In other words, the center of mass of the body is not necessarily at rest with respect to the observers who attribute zero spatial momentum to the body [11];

(iii) With respect to $z(\tau)$ and $n^{a}(\tau)\left(\equiv n^{a}\right.$ at $\left.z(\tau)\right)$, the total angular momentum of the body (also properly defined in Ref. [4]) is characterized by a spin fourvector $S^{a}(\tau)$ orthogonal to $p^{a}(\tau) \equiv p^{a}(z(\tau), n(\tau))$;

(iv) Along $z(\tau)$, the four-momentum $p^{a}(\tau)$ and the spin $S^{a}(\tau)$ change according to the coupled equations

$$
\begin{aligned}
\frac{D p^{a}}{d \tau} & =\frac{1}{2} S^{b c} v^{d} R_{b c d}{ }^{a}+F^{a}, \\
\frac{D S^{a b}}{d \tau} & =\left(p^{a} v^{b}-v^{a} p^{b}\right)+G^{a b}
\end{aligned}
$$

where $D / d \tau:=v^{b} \nabla_{b}$ is the covariant derivative operator along $z(\tau), S^{a b}=\epsilon^{a b c d} S_{c} n_{d}\left(\epsilon_{a b c d}\right.$ is the totally antisymmetric, Levi-Civita pseudo tensor), and $F^{a}$ and $G^{a b}$ are force-like [20] and torquelike terms which can be expanded in terms of couplings between the Riemann curvature tensor $R_{a b c}{ }^{d}$ (and its derivatives) and higher multipole moments (quadrupole, octupole, and so on) of $T^{a b}$ (with respect to $z(\tau)$ and $\left.n^{a}(\tau)\right)$. For now, their exact forms are not important;

(v) For each (if there is any) symmetry of the spacetime with generator $\xi^{a}$ (which thus satisfies $\nabla_{a} \xi_{b}+$ $\left.\nabla_{b} \xi_{a}=0\right)$, there is an associated constraint between $F^{a}$ and $G^{a b}$ given by

$$
F^{a} \xi_{a}+\frac{1}{2} G^{a b} \nabla_{a} \xi_{b}=0
$$

(Noting that $G^{a b}$ is antisymmetric.)

These (together with the precise definitions of $p^{a}, S^{a b}$, center of mass, $F^{a}$, and $G^{a b}$ ) are the main results of the extensive analysis performed by Dixon.

One immediate consequence concerns maximallysymmetric (Minkowski, de Sitter, and anti-de Sitter) spacetimes. In such spaces, the number of symmetries is enough to make $\xi_{a}$ and $\nabla_{a} \xi_{b}$ completely independent at each point. Therefore, the number of constraints given in (v) completely determines $F^{a}=0$ and $G^{a b}=0$. This, in turn, implies (see Ref. [4]): (a) $p^{a}(\tau)$ is parallel to $v^{a}(\tau)$, with proportionality factor $m(\tau)$ being constant (interpreted as the rest mass of the system), (b) the spin $S^{a}(\tau)$ is conserved, and, more importantly, (c) $v^{a}(\tau)$ is parallel-transported along itself. In other words, the center of mass of the body follows an exact geodesic of the spacetime. No swimming is possible in maximallysymmetric spacetimes, contradicting the result obtained in Eq. (5).

This proves, beyond doubt, that the original analysis of Ref. [1] is inappropriate to investigate the motion of extended bodies in curved spacetime, even in the regime where its approximations are valid. Considering that this kind of analysis stands as the sole basis of the swimming effect to date, this would seem to drown the hopes of any spacetime swimmer.

\section{RESCUING WISDOM'S TRIPOD IN SCHWARZSCHILD SPACETIME}

Notwithstanding the negative general result in maximally-symmetric spacetimes, let us take a closer look at Eqs. (677). As already mentioned, the forcelike and torque-like terms, $F^{a}$ and $G^{a b}$, depend on the coupling between the geometry of the spacetime and the multipole moments of $T^{a b}$. Under the same assumptions made in Ref. 1] concerning length scales of the body being much smaller than length scales introduced by the curvature of the spacetime, the dominant contribution to $F^{a}$ and $G^{a b}$ comes, in general, from the quadrupole moment of $T^{a b}$ :

$$
\begin{aligned}
F^{a}= & -\frac{1}{6} J^{b c d e} \nabla^{a} R_{b c d e} \\
& +\left(\begin{array}{c}
\text { higher-multipole, } \\
\text { higher-curvature-derivative } \\
\text { terms }
\end{array}\right) \\
G^{a b}= & \frac{4}{3} R^{[a} c d e^{J^{b] c d e}} \\
& +\left(\begin{array}{c}
\text { higher-multipole, } \\
\text { higher-curvature-derivative } \\
\text { terms }
\end{array}\right)
\end{aligned}
$$

where $J^{a b c d}$ is the reduced quadrupole moment of $T^{a b}$, defined in Refs. [4, 6], calculated in the zero-spatialmomentum frame and with respect to the center of mass. (Brackets stand for antisymmetrization over the enclosed indices: $A^{[a b]}:=\left(A^{a b}-A^{b a}\right) / 2$.) The exact expression for $J^{a b c d}$ in terms of $T^{a b}$ (and $z(\tau)$ ) does not concern us now. It suffices to mention a few facts. First, it has the same index symmetries as the Riemann curvature tensor $R^{a b c d}$ (therefore, only 20 independent components instead of the 60 expected - hence the term "reduced"). Second - and here is where all the intricate definitions of Refs. [4, 6] pay off -, the covariant conservation of $T^{a b}$ imposes no (algebraic or differential) constraints on $J^{a b c d}$ nor on any of the higher reduced multipole moments, for that matter. Therefore, at least in principle, $J^{a b c d}$ can be independently assigned as a function of the propertime $\tau$, reflecting conformational changes prescribed for the body in its own rest frame, and then Eqs. (667) (ten components in total) can be integrated to determine the evolution of $p^{a}, S^{a}$, and $v^{a}$ (ten components in total) and, thus, the world-line $z(\tau)$ itself. Such is the power of Dixon's formalism.

Now, let us apply this machinery to Wisdom's tripod in Schwarzschild spacetime. Right from the start, the symmetry of the setup vanishes most of the components 
of a tensor with the index symmetries of $J^{a b c d}$, except (in an orthonormal basis $\left\{e_{0}^{a}, e_{1}^{a}, e_{2}^{a}, e_{3}^{a}\right\}$ aligned with the coordinates $\{t, r, \theta, \phi\}$, respectively): $J^{0101}, J^{0202}=J^{0303}$, $J^{0212}=J^{0313}, J^{1212}=J^{1313}$, and $J^{2323}$. Combining this with the Riemann curvature tensor, one finds that the quadrupole contribution to the torque in Eq. (10) vanishes: $R_{c d e}^{[a} J^{b] c d e}=0$. This, in turn, adds complication to the analysis of Wisdom's tripod because, then, in order to calculate $G^{a b}$, one must look at the next order, which couples the (reduced) octupole moment $J^{\text {abcde }}$ of its stress-energy-momentum tensor (whose typical order of magnitude is $m c^{2} l^{3}$, where $m$ is the mass of the tripod and $l$ its typical linear size) to the covariant derivative of the curvature tensor $\left(\sim G M /\left(c^{2} r^{4}\right)\right.$, where $r$ is the radial coordinate of the center of mass of the tripod). In spite of its intricate expression - see Eqs. (13.8,13.9,A1.2,5.35) of Ref. 6- -, in the end the symmetries of the system and of $J^{a b c d e}$ (see Eqs. (5.33,5.36) of Ref. [6]) lead, to zeroth-order in $\dot{r} / c$ [21], to

$$
G^{01} \approx \frac{4 G M}{c^{2} r^{4}} J^{22110}
$$

with all other components of $G^{a b}$ vanishing. In addition, we note that the symmetry of the setup also imposes $S^{a}=0=S^{a b}$, which forces $v^{a}$ and $p^{a}$ to be slightly misaligned in order for the right-hand side of Eq. (7) to vanish. To first order in both $G M /\left(c^{2} r\right)$ and $v^{1} / c=$ $\dot{r} / c$ [22],

$$
p^{a} \approx m v^{a}+\frac{4 G M}{c^{3} r^{4}} J^{22110} e_{1}^{a}
$$

Turning attention to the force-like term, Eq. (9), the symmetries of $J^{a b c d}$ combined with the Riemann curvature tensor lead to

$$
F^{1}=-\frac{4 G M}{c^{2} r^{4}}\left(J^{0101}-J^{0202}+J^{1212}-J^{2323}\right),
$$

with all other components of $F^{a}$ vanishing. Therefore, substituting Eqs. (1213) and $S^{a b}=0$ into Eq. (6), we have, to first order in both $G M /\left(c^{2} r\right)$ and $\dot{r} / c$,

$$
\frac{D\left(m v^{a}\right)}{d \tau} \approx-\frac{4 G M \Lambda}{c^{2} r^{4}} e_{1}^{a},
$$

where $\Lambda=J^{0101}-J^{0202}+J^{1212}-J^{2323}+\dot{J}^{22110} / c$, which is of order $m c^{2} l^{2}$. This equation of motion tells us how much the tripod's center of mass can accelerate (in the nonrelativistic regime) with respect to exact geodesic motion simply due to the fact that, being extended, it experiences an inhomogeneous gravitational field (more properly, inhomogeneous tidal effect). This is not swimming but one can use this estimate to obtain a stringent upper bound on the order of magnitude of the swimming effect for Wisdom's setup. In particular, no variation in the quadrupole and octupole moments of $T^{a b}$, in the regime assumed in Ref. [1], can change the $r^{-4}$ dependence of the right-hand side of Eq. (14) to a less-negative power. Therefore, this simple order-of-magnitude estimate is enough to prove that Eq. (2) cannot hold true.

The reduced quadrupole and octupole moments of the tripod, needed to determine $\Lambda$, could, in principle, be given as a function of $\tau$ [23]. But more realistically, these moments should be calculated from the prescribed internal motion of the tripod. In other words, they must be given in terms of $l$ and $\alpha$; not only through their instantaneous values $l(\tau)$ and $\alpha(\tau)$ but also dependent on the instantaneous values of $\dot{l}(\tau), \dot{\alpha}(\tau), \ddot{l}(\tau)$, and $\ddot{\alpha}(\tau)$. The $(\dot{l}, \dot{\alpha})$-dependence can come in due to possible energy flows inside the body as its conformation changes, while $(\dot{l}, \dot{\alpha}, \ddot{l}, \ddot{\alpha})$-dependence comes in due to $i n$ ternal forces (stresses) necessary to accelerate its different parts with respect to the center of mass. Recall that $T^{a b}$ does, indeed, contain all this information.

Comparing two slightly different prescriptions for the time dependence of $l$ and $\alpha$ (differing by $\delta l(\tau)$ and $\delta \alpha(\tau)$, respectively), we can expand the associated differences in the quadrupole and octupole moments to first order in $\delta l$ and $\delta \alpha$ (and their derivatives) and, eventually, express the change in $\Lambda$ as

$$
\delta \Lambda=\sum_{q \in \mathcal{I}} \frac{\partial \Lambda}{\partial q} \delta q(\tau)
$$

where $\mathcal{I}=\{l, \alpha, \dot{l}, \dot{\alpha}, \ddot{l}, \ddot{\alpha}\} 24$. Then, from here on, the analysis follows as in Ref. [1]. Using Eq. (15) in Eq. (14) and working in the same regime as Ref. 1] [see below Eq. (2)], the following approximate result holds in the limit of short periods of time [25]:

$$
m \delta r \approx-\frac{4 G M}{c^{2} r^{4}}\left(\frac{\partial \Lambda}{\partial \ddot{l}} \delta l+\frac{\partial \Lambda}{\partial \ddot{\alpha}} \delta \alpha\right) .
$$

Applying this result to the sequence of deformations (1), a non-zero net deviation in the radial position of the center of mass of the tripod, at the end of each cycle, only arises if the right-hand side of Eq. (16) is not an exact differential in the parameter space $(l, \alpha)$. In that case, it is given by

$$
\delta r \approx-\left.\frac{4 G M}{m c^{2} r^{4}}\left(\frac{\partial^{2} \Lambda}{\partial \alpha \partial \ddot{l}}-\frac{\partial^{2} \Lambda}{\partial l \partial \ddot{\alpha}}\right)\right|_{\left(l_{0}, \alpha_{0}\right)} \delta l \delta \alpha .
$$

In order to proceed beyond this point, we must calculate explicitly the relevant quadrupole and octupole components which contribute to $\Lambda$. More precisely, we only need those quadrupole components which carry dependence on $\ddot{l}$ or $\ddot{\alpha}$ and the octupole components which depend on $\dot{l}, \dot{\alpha}, \ddot{l}$ or $\ddot{\alpha}$. Splitting the stress-energymomentum tensor of the tripod as $T^{a b}=T_{(m)}^{a b}+T_{(\ell)}^{a b}$, with $T_{(m)}^{a b}$ and $T_{(\ell)}^{a b}$ separately describing the point masses and the legs, respectively, only $T_{(\ell)}^{a b}$ shows explicit dependence on $\ddot{l}$ or $\ddot{\alpha}$. This can be easily understood considering that the legs are responsible for transmitting the inner forces which ensure the prescribed changes in the relative positions of the point masses. On the other hand, modeling 
the legs as ideal rods (i.e., rods whose total masses and momenta can be neglected in the low-velocity regime so that they transmit forces and torques integrally), one can verify that only $T_{(m)}^{a b}$ contribute to $\dot{J}^{22110}$ to leading order - due to the momentum-density distribution. Hence, although the point masses of the tripod dominate, through their mass distribution, the value of $\Lambda$ - which determines how much the tripod deviate from geodesic motion for being extended -, it is the coupling of the spacetime curvature to (moments of) momenta and stresses - an inherent feature of relativistic gravity theories - which impel the tripod to swim in this scenario.

For concreteness sake, considering only leading-order curvature terms in expression (17) and modeling the tripod's legs as ideal rods (in the sense defined above), we can compute the relevant components of $J^{a b c d}$ and $J^{\text {abcde }}$, which are $J_{(\ell)}^{1212}, J_{(\ell)}^{2323}$ and $J_{(m)}^{22110}$, as in flat space (see Refs. [4, 6] ):

$$
\begin{aligned}
J_{(\ell)}^{i j i j} & =\frac{1}{4} \int d^{3} x\left[x^{i} x^{i} T_{(\ell)}^{j j}+x^{j} x^{j} T_{(\ell)}^{i i}-2 x^{i} x^{j} T_{(\ell)}^{i j}\right] \\
J_{(m)}^{i i j j 0} & =\frac{1}{8} \int d^{3} x x^{i} x^{j}\left[x^{i} T_{(m)}^{j 0}-x^{j} T_{(m)}^{i 0}\right]
\end{aligned}
$$

where $\left\{x^{i}\right\}_{i=1,2,3}$ is a (local) Cartesian coordinate system whose origin is located at the center of mass of the tripod and with $x^{1}$ aligned with the radial direction. The evaluation of $J_{(\ell)}^{1212}$ and $J_{(\ell)}^{2323}$ depends on how the inner forces, leading to a given sequence of conformational changes, are exerted. In particularly simple realizations, in which each leg is subject only to forces in the plane containing the leg and the tripod's symmetry axis, one obtains $J_{(\ell)}^{2323}=0$ and the contribution coming from $J_{(\ell)}^{1212}$ and $J_{(m)}^{22110}$ leads to

$$
\delta r \approx-\frac{G M}{c^{2} r}\left(\frac{l_{0}}{r}\right)^{3} f\left(k, \alpha_{0}\right) \sin \left(2 \alpha_{0}\right) \delta l \delta \alpha,
$$

where $k:=3 m_{1} /\left(m_{0}+3 m_{1}\right)$ and $f\left(k, \alpha_{0}\right)$ is a dimensionless function whose exact expression still depends on further details of how these forces are exerted on each leg [26]. This result - and, more generally, Eq. (17) replaces the one given in Eq. (2) and it shows that in the scenario analyzed in Ref. 1] swimming is a much subtler effect. In fact, for a one-meter-long tripod near Earth's surface, swimming is suppressed by a $10^{-7}$ factor in comparison to Eq. (2).

\section{DISCUSSION: THE CURVED-SPACETIME SWIMMING EFFECT}

In Ref. [1], Wisdom made use of a classical-mechanics analogue - that of an articulated, varying-length bipod moving on the surface of a 2 -sphere without external tangential forces - in order to ingeniously motivate the idea of swimming in curved spaces. There, the "swimming" is indeed of order $(l / r)^{2}$, where $r$ is the radius of the 2sphere. (This unfortunate similarity with Eq. (2) may explain why the latter stood undisputed for so long.) It is interesting to point out that Dixon's formalism (with some possibly minor changes to account for lower dimensionality) can be applied to this classical-mechanics system, accounting for the same order- $(l / r)^{2}$ effect. But differently than what occurs for the tripod in the scenario analyzed in Ref. [1], it is the quadrupole contribution to the torque-like term $G^{i 0} \propto R^{[i}{ }_{c d e} J^{0] c d e}$ in Eq. (7) which propels the center of mass of the bipod along the 2 -sphere. Due to symmetries of the body and of the spacetime (which can be seen as the $1+2$ Einstein static universe), this torque-like term cannot induce rotation on the bipod; instead, it is cancelled by the "misalignment" term $p^{i} v^{0}-v^{i} p^{0}$. As a result, at each cycle, the center of mass of the bipod is propelled along the 2-sphere $\left(v^{i} \neq 0\right)$ while its total spatial momentum is kept null (for $F^{a}=0$ due to the constraints imposed by the geometric symmetries via Eq. (8) ). Interestingly enough, a similar conclusion holds for a bipod in $1+3$ Einstein static universe.

The application of Dixon's formalism to the bipod on the 2-sphere serves more than simply as a consistency check: it also shows that swimming in curved spacetimes can be of the same order of magnitude as anticipated by Wisdom. For instance, it is quite possible that Wisdom's tripod in Schwarzschild spacetime can swim at order $G M l^{2} \delta l \delta \alpha /\left(c^{2} r^{3}\right)$ in scenarios where $G^{a b}=R^{[a}{ }_{c d e} J^{b] c d e} \neq 0$ in Eq. (7) or $S^{a b} \neq 0$ in Eq. (6) - perhaps in orbital motion. Be it as it may, one should use Dixon's covariant, local formalism, summarized in Eqs. (6]7/9[10), in order not to arrive at fictitious effects and also to identify body shapes and internal cyclic motions which might lead to optimal swimmers in a given spacetime.

\section{Acknowledgments}

The authors acknowledge full (R. S.) and partial (G. M. and D. V.) financial support from São Paulo Research Foundation (FAPESP) under Grants No. 2015/10373-4, 2015/22482-2, and 2013/12165-4, respectively. G. M. also acknowledges partial financial support from Conselho Nacional de Desenvolvimento Científico e Tecnológico (CNPq). 
[1] J. Wisdom, Science, 299, 1865 (2003).

[2] J. Wisdom, Phys. Today, 56, 12 (2003).

[3] G. A. Landis, Phys. Today 56, 12 (2003).

[4] W. G. Dixon, Proc. Roy. Soc. Lond. A 314, 499 (1970).

[5] W. G. Dixon, Proc. Roy. Soc. Lond. A 319, 509 (1970).

[6] W. G. Dixon, Philos. Trans. Roy. Soc. Lond. A 277, 59 (1974).

[7] W. G. Dixon, Gen. Rel. Grav. 4, 199 (1973).

[8] Dixon's analysis is actually more general than this, allowing for the presence of external electromagnetic forces.

[9] It is assumed that the spatial extension of the body is finite and such that the convex hull $\mathcal{B}$ of the intersection of its "world-tube" (i.e., the set of spacetime points where $\left.T^{a b} \neq 0\right)$ with any space-like hypersurface $\Sigma$ is contained in a normal neighborhood of any point of $\mathcal{B}$. This means that there is only one geodesic, in $\mathcal{B}$, connecting any two spatially-separated points of the body. Moreover, wherever $T^{a b} \neq 0$, the momentum density is assumed to be time-like and future-directed according to all observers; i.e., $T_{a b} n^{a} n^{b}>0$ and $T_{a b} T^{b}{ }_{c} n^{a} n^{c}<0$ for all time-like vectors $n^{a}$. Finally, these conditions are supposed to hold also for the momentum density "propagated" from any point $x$ to any point $x^{\prime}$, both in $\mathcal{B}$, where the "propagation" is performed along the geodesic connecting $x$ and $x^{\prime}$, according to maps $K_{b}^{a^{\prime}}$ and $H_{b}^{a^{\prime}}$ which are properly defined in Refs. [4, 6].

[10] W. Beiglböck, Commun. Math. Phys. 5, 106 (1967).

[11] This already occurs in special relativity but only in the presence of external forces. This is related to what has been termed - somewhat unfortunately - hidden momentum [12] and caused much confusion recently. See, e.g., Refs. 13 19].

[12] W. Shockley and R. P. James, Phys. Rev. Lett. 18, 876 (1967).

[13] M. Mansuripur, Phys. Rev. Lett. 108, 193901 (2012).

[14] A. Cho, Science 336, 404 (2012).

[15] D. A. T. Vanzella, Phys. Rev. Lett. 110, 089401 (2013).
[16] S. M. Barnett, Phys. Rev. Lett. 110, 089402 (2013).

[17] P. L. Saldanha, Phys. Rev. Lett. 110, 089403 (2013).

[18] M. Khorrami, Phys. Rev. Lett. 110, 089404 (2013).

[19] M. Mansuripur, Phys. Rev. Lett. 110, 089405 (2013).

[20] Here we chose to separate the term linear in $S^{a b}$ from $F^{a}$ defined in Refs. [4, 6] because of the simpler form assumed by the constraint in (v). But it is worth mentioning that the first term on the right-hand side of Eq. (6) actually accounts for the net tidal force acting on the extended body.

[21] This is enough for keeping the equation of motion (14) correct to first order in $G M /\left(c^{2} r\right)$.

[22] Recall that, to first order in $\dot{r} / c, v^{a}=c e_{0}^{a}+\dot{r} e_{1}^{a}$.

[23] Which in this nonrelativistic, small-GM/( $\left.c^{2} r\right)$ regime is the same as $t$.

[24] As will be made explicit later on, the octupole component $J^{22110}$ is dominated by the momentum-density distribution of the tripod - see Eq. (19). This makes $J^{22110}$ independent of $\ddot{l}$ and $\ddot{\alpha}$ to leading order, which, in turn, makes $\Lambda$ independent of third-order time derivatives of $l$ and $\alpha$ in the regime considered here.

[25] The validity of this approximate expression is actually what defines "short periods of time." Typically, Eq. (16) is a valid approximation as long as $\delta l$ and $\delta \alpha$ are variations taking place on a time scale $\delta t$ much smaller than $l / c$. Obviously, this demands that the conformational changes of the tripod be pre-programmed along its extension, for there would be no time for a central signal to propagate and orchestrate such changes. Moreover, the "small-velocity regime" for the variation of $l$ and $\alpha$ also imposes $\delta l, l \delta \alpha \ll c \delta t(\ll l)$. These limitations also apply to the treatment of Ref. [1].

[26] In one particular realization, for instance, $f\left(k, \alpha_{0}\right)=$ $k\left[1 / 2+k-3 k^{2} / 2+3 k^{3} / 16+(k-2) k^{2} \cos \left(2 \alpha_{0}\right) / 4+\right.$ $\left.k^{3} \cos \left(4 \alpha_{0}\right) / 16\right]$. 